\title{
Important clinical lesson in a patent Vitello-intestinal duct in a 3 year old male: Literature review and a case report.
}

\author{
*Dr. Fente B. G. BMBCH, FWACS, FMCS, FICS: *Dr. Tabowei BJ MBBS, \\ FMCS, **DR. Kombo BB MBBS, FRCS, FMCS \\ * Department of Surgery Niger Delta University Teaching Hospital, Okolobiri, Bayelsa State, Nigeria. \\ **Department of Surgery, University of Port Harcourt Teaching Hospital, Port Harcourt, Rivers State, Nigeria
}

\begin{abstract}
During the 3rd week of intrauterine life there is a communication between the intraembryonic gut and the yolk sac. As the development proceeds this communication narrows into a tube known as the vitellointestinal duct (VID). With the establishment of placental nutrition this duct usually becomes obliterated by the end of the 7th week of intrauterine life. In about $2 \%$ of humans this duct persists and gives rise to a group of anomalies of which Meckel's diverticulum is the commonest and complete patency of the duct is the rarest. We report a case of a 3 year old male who had umbilical discharge from birth and surgical intervention done without proper diagnostic procedures. High index of suspicion is an important clinical lesson that should be applied in the management of rare cases.
\end{abstract}

\section{Introduction}

Patent Vitello-intestinal Duct (PVID) results from failed obliteration of the fetal omphalocele coelom (herniated loops of intestine in the umbilical cord) during the development of the midgut ${ }^{1,2}$. During the $3 \mathrm{rd}$ week of intrauterine life there is a communication between the intraembryonic gut and the yolk sac. As the development proceeds this communication narrows into a tube known as the vitello-intestinal duct (VID). With the establishment of placental nutrition this duct usually becomes obliterated by the end of the 7th week of intrauterine life. At approximately the 10th week of gestation, the midgut begins its return into the abdominal cavity ${ }^{1-3}$. This return occurs by a highly complex developmental process, and as a result, numerous anomalies of the bowel may ensue ${ }^{3}$. These include bowel atresias and stenoses, abnormalities of the vitellointestinal duct, failure of caecal descent, malrotation, malfixation, reversed bowel rotation and exomphalos ${ }^{1-3}$. In about $2 \%$ of humans this duct persists and gives rise to a group of anomalies of which Meckel's diverticulum is the commonest and complete patency of the duct is the rarest ${ }^{1,2}$.

Remnants of the vitello-intestinal duct are said to be present in 2 to $4 \%$ of all routine postmortem examinations ${ }^{2}$, but presumably many people live their allotted span of life despite their presence and at no time have symptoms referable to them ${ }^{2,3}$. On the other hand, these vestigial structures may make their presence known dramatically in the first few years of life, or, more rarely, in adult life $\mathrm{f}^{2-4}$. The complications to which they are subject are serious and are commonest in infants and young children of the male sex ${ }^{2}$.

A patent vitello-intestinal duct (VID) can present as discharging umbilical sinus, umbilical nodule or polyp, bleeding from intestinal mucosa and Intestinal small bowel prolapsed ${ }^{2-7}$. There may be associated cellulitis, which in rare cases can rapidly progress to necrotizing fasciitis and severe sepsis ${ }^{4,5}$. The differential diagnosis of persistent umbilical discharge includes a patent urachus (a congenital communication with the bladder), an incarcerated hernia, metastatic disease, tuberculosis or some other chronic infection ${ }^{4,5}$. At 11 months of age the differential usually rests between a patient VID and urachus ${ }^{4,5}$.

Various surgical operative interventions have been described for the patent VID. The umbilical approach to VID has been reported through one of the three approaches: a circular incision around the umbilicus with excision of umbilicus and umbilicoplasty ${ }^{2}$, infraumblical incision without umbilical excision so the patient retains the umblicus, 5 , a circular incision around umbilicus without umbilical reconstruction, hence the patient losses the umbilicus ${ }^{7}$. These approaches allow the patent VID to be mobilized and then a decision can be made as to how the patent VID is to be resected. These options for resection are either a wedge a segmental small bowel resection. In this case, the approach used preserved the umbilicus rendering the need for umbilical reconstruction.

\section{Case Presentation}

This 3 years old male child had umbilical discharge since birth. On the tenth day of life when the umbilical cord fell off, the parents noticed raised pink edges of the umbilicus discharging small quantities of sticky greenish material. In some occasion, the umbilical discharges were pus or faecal matter. Baby had been on treatment with native herbs and drugs bought from chemist but umbilical discharge persisted. Meanwhile baby took feeds and passed urine and stools normally. But as patient was said to have had fever and pussy 
umbilical discharge and was taken to a private clinic where the finding were said to have suggested abscess and incision/drainage was done. As faecal material and intestine were seen patient was referred to us.

Past history revealed that he was delivered at tern per vagina. Delivery was conducted by traditional birth attendant. Antenatal period was said to be uneventful though unmonitored. Baby cried immediately after birth.

Clinical examination revealed a healthy child with faecal matter discharging from the umbilical area. Polydactily of the right hand (Figs $1 \& 2$ ) with no other congenital anomalies was noted.

Provisional diagnosis of arthrogenic small bowel injury in a child with possible patent vitello-intestine duct (PVID) was made. After resuscitations with fluid and routine preoperative investigations protocol of FBC and cross matching of blood, urgent exploration laparatomy was done.

\section{SURGICAL PROCEDURE:}

Laparatomy under general anesthesia was done using lower midline incision. At laparatomy, the outer surface of the emerging loop was found to be firmly adherent to all the layers of the anterior abdominal wall (Figure 2). It was dissected from the abdominal wall layers. Intraoperative findings are shown pictorially as Figures 3-6. After complete reduction (Figures $5 \& 6$ ) a defect of $1.4 \times 1.4$ centimeters was found in the small intestine (Figure 2) at the point of adherence with the abdominal wall suggesting its patency with external environment through the umbilicus.

We diagnosed the case as patent vitellointestinal duct. Primary resection of the small intestine shown in figures $5 \& 6$ and end to end anastomosis was done. The defect of PVID over the abdominal wall was primarily closed and the umbilicus was reconstructed. Primary closure of the abdominal wound was done.

Patient had smooth and uneventful recovery and was discharged to the outpatient clinic for follow up.

\section{Discussion}

The incidence of a completely patent VID is reported to be $0.0063-0.067 \%{ }^{3}$. Of all the anomalies of the VID, complete patency of the duct is the rarest ${ }^{3}$. Failure of obliteration of the embryonic vitellointestinal duct leads to various congenital anomalies like - Meckel's diverticulum, vitelline cord, enteric cyst, umbilical sinus, enteric fistula or hemorrhagic umbilical mass ${ }^{1-3}$. Patient may present because of the anomaly itself or due to complications secondary to the anomalies like intestinal obstruction due to volvulus, intussusception or adhesions $^{1-7}$. Totally PVID is a very rare anomaly and very few cases were reported in the literature ${ }^{2,3}$. This single case of a patent Vitello-intestinal duct in a 3 year old male is the first seen by us after 30 years of practice in this part of the Niger Delta. This is an indication of its rareness. Patent vitellointestinal duct may present itself as continuous or intermittent discharge through the umbilicus. This case presented with intermittent discharge.

Primary closure of the VID at the neonatal period is possible if the patient arrives early without any complications. Where the defect is large as in this case, resection of the loop of intestine near the patent duct followed by primary anastomosis is the procedure of choice ${ }^{2-4}$.This was done in this case.

If the patient presents later in life as in this case with additional congenital malformation, in this case, Polydactly, a high index of suspicion should be applied before any surgical intervention be done. Ultrasound examination of the abdomen for intraabdomenal collections/other congenital anomalies and water soluble contrast injecting through the opening to show the dye in the intestine are investigations that need to be done before any intervention. None of these were done before the intervention of incision and drainage was tried at the private clinic.

\section{Conclusion}

Patent VID with continuous or intermittent discharge is a very rare condition in our environment. This is the first case we have seen. It needs prompt diagnosis, surgical intervention and repair of the defect. , A high index of suspicion and investigations are necessary before any surgical intervention in cases of late presentation.

\section{References:}

[1]. Peter IW, Patrica C. Embryology and development.Gray’s Anatomy Churchill Livingstone, $38^{\text {th }}$ edition: 1995. 188-190.

[2]. Agrawal S, Memon A. Patent vitello-intestinal duct. BMJ Case Reports 2010;10.1136/bcr.12.2009.2594,

[3]. Prashant NM, Ashok MB, Virsing PH, Jitendra HM: Patent vitellointestinal duct with prolapse of inverted loop of small intestine: a case report Journal of Medical Case Reports 2007, 1:49 doi:10.1186/1752-1947-1-49

[4]. Giacalone JP, Vanrylcel F Belva. Surgical Treatment of Patent Omphalomesenteric Duct presenting as Fecal Umbilical Discharge. Acta ehir Belg. 2004;10: 211-213.

[5]. Sheth NP. Transumblical Resection and Umbilical Plasty for Patent Omphaloesenteric Duct. Pediatric Surg Int. 2000; $16: 152$.

[6]. Fleming F, Ishtiaq A, O'Connor J. Patent Omphalomesenteric Duct Presenting as an Umbilical Discharge. Ir Med Journal. 1994; 94:182.

[7]. Elebute EA, Ransome-Kuti O. Patent Vitello-intestinal Duct with Ileal Prolapse. Arch Surg. 1965; 91:456-60.

FIGURE 1: POLYDACTLY OF RIGHT HAND 


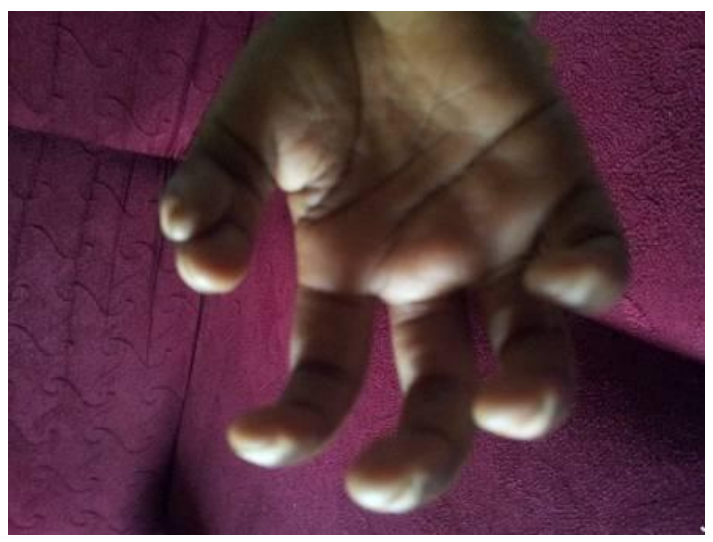

Figure 2 The bowel is shown connected through the duct.

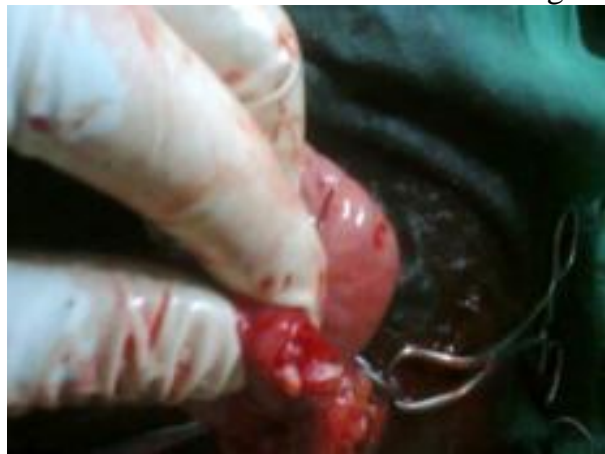

Figure 3 The bowel is shown through the mesenteric opening it passed to the umblicus

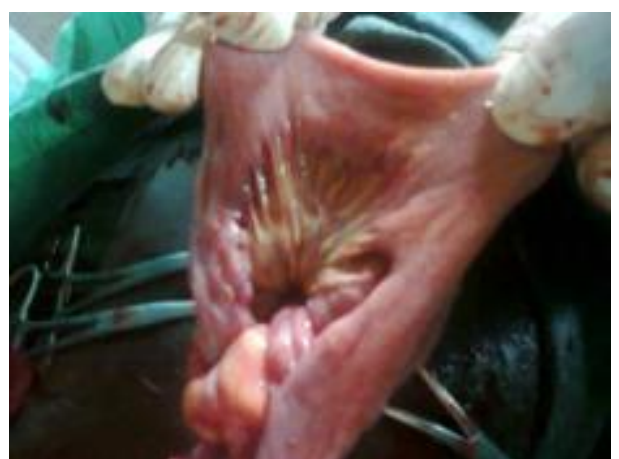

Figure 4 Disection to free the patent vittello-intestinal duct.

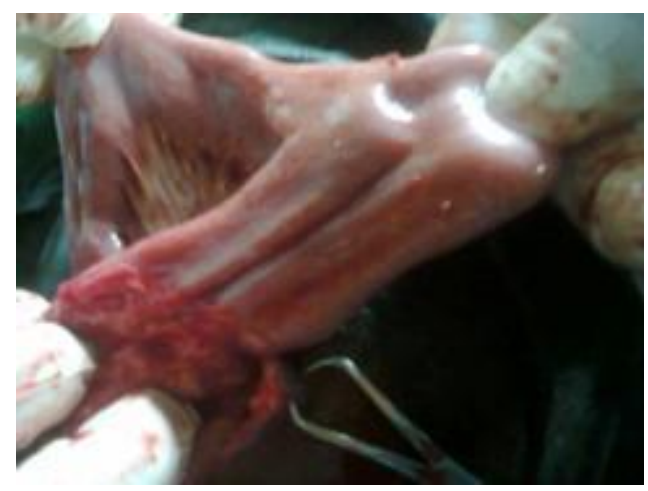

Figure 5 stem of the ' $Y$ ' shaped loop fixed to the anterior abdominal wall. 


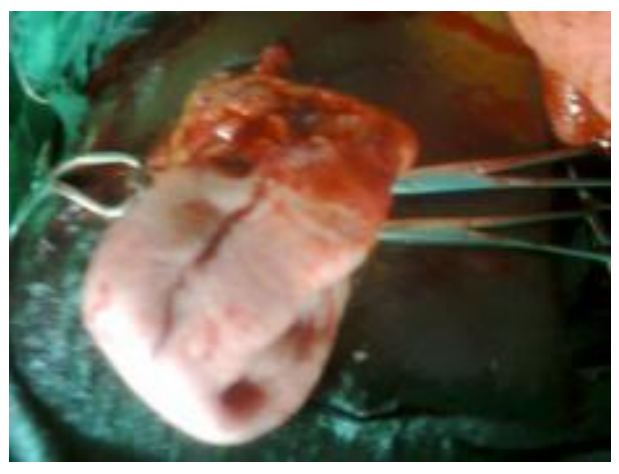

Figure 6 Bowel when freed to show area resected

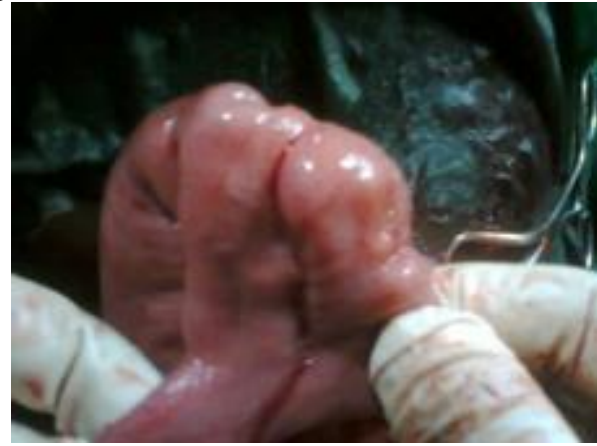

Figure 7 Healed Laparatomy scar at discharge

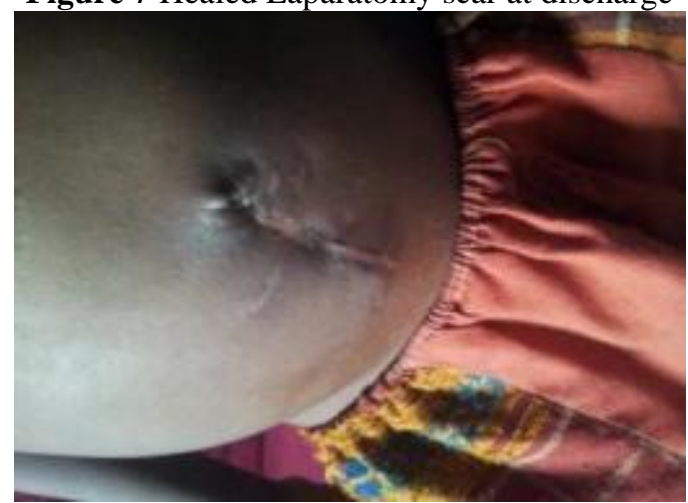

